\title{
Evidence of Homoclinic Chaos in the Plasma of a Glow Discharge
}

\author{
Thomas Braun, (1) Jorge A. Lisboa, (1), (2) and Jason A. C. Gallas (1),(3),(4), (a) \\ ${ }^{(1)}$ Instituto de Física da Universidad Federal do Rio Grande do Sul, 90000 Porto Alegre, Rio Grande do Sul, Brazil \\ ${ }^{(2)}$ Departamento de Engenharia Elétrica da Universidad Federal do Rio Grande do Sul. \\ 90000 Porto Alegre, Rio Grande do Sul, Brazil \\ ${ }^{(3)}$ Laboratório de Óptica Quântica da Universidade Federal de Santa Catarina, \\ Departamento de Física, 88049 Florianópolis, Santa Catarina, Brazil \\ ${ }^{(4)}$ Höchstleistungsrechenzentrum (HLRZ), Kernforschungsanlage Jülich, W-5170 Jülich, Germany
}

(Received 16 December 1991)

\begin{abstract}
Homoclinic chaos is shown to occur in the electric current measured on an electrical discharge in argon. We report a clear sequence of four hesitations followed by a reverse period doubling. The experimental signals are used to construct return-time and time-of-flight maps that evidence a Shilnikov scenario in the system.
\end{abstract}

PACS numbers: $52.35 . P y, 52.80 . \mathrm{Hc}$

Apart from the most known routes to chaos like period doubling, intermittency, quasiperiodicity, etc. [1], different transition sequences from periodic to chaotic patterns have also been observed. An example of such are the so-called alternating periodic-chaotic (APC) sequences [2]. As the name indicates, these sequences consist of an alternation of periodic-chaotic states of largeand small-amplitude oscillations (mixed-mode oscillations) obtained when there is a change of a control parameter of the system. Typically, a periodic state is composed of one large-amplitude peak followed by $n$ smallamplitude peaks per period, referred to as a $P^{(n)}$ pattern. Periodic states in an APC sequence are a succession of $P^{(n)}$ patterns where $n=0,1,2, \ldots$ (but finite). Between two $P^{(n)}$ and $P^{(n+1)}$ periodic states there is always a chaotic state $C^{(n)}$ formed by a nonperiodic (random) mixture of states having $P^{(n)}$ and $P^{(n+1)}$ patterns (occasionally, but with much less frequency, having $P^{(n-1)}$ and $P^{(n+2)}$ patterns in the mixture as well). The system is then said to "hesitate" between $P^{(n)}$ and $P^{(n+1)}$. A chaotic $C^{(n)}$ pattern is normally reached from a periodic $P^{(n)}$ pattern through a period doubling cascade. Much of the behavior concerning APC sequences is understood in terms of a homoclinic chaos scenario. A key point in this scenario is the presence of a homoclinic orbit in the phase space. Assuming the presence of an invariant set in phase space (e.g., a fixed point or a periodic orbit), if there is an orbit approaching the invariant set asymptotically for $t \rightarrow \pm \infty$, this orbit is said to be homoclinic to the invariant set [3]. Here we concentrate on invariant sets of saddle type so that trajectories in phase space approach the invariant set along its stable manifold and leave it along the unstable manifold.

Homoclinic orbits are associated with erratic behavior (chaos) in a dynamical system [3,4]. Experimentally it is quite difficult to exactly reach homoclinic orbits because of unavoidable small fluctuations (noise). Close to them chaotic behavior might be expected. This elusive behavior was previously experimentally observed in at least two situations: in the Belousov-Zhabotinskii reaction [5] and, shortly later, in the intensity of a laser with a saturable absorber [6] and of a laser with an electro-optic modula- tor [7]. The theoretical background for these observations is provided by works developed by Shilnikov and co-workers in which the homoclinicity to a saddle focus [8] and to a saddle cycle [9] was analyzed. Within the scenario described by Shilnikov, homoclinic orbits might not only be at the origin of chaotic behavior but can also generate regular bifurcation sequences such as, for instance, the APC sequence. This was theoretically seen in both cases, i.e., when the invariant set to which the orbit is homoclinic is either a saddle focus [10] or a saddle cycle [1 1].

We report experimental observations of mixed-mode oscillations displaying APC sequences which are explained in terms of homoclinic chaos. Our experimental signals are obtained from a dc-excited glow discharge similar to the one where deterministic chaos was first observed in plasmas [12]. Recall that definitive observations of deterministic chaos in plasmas are rare [12,13]. The evidences reported in this paper enable us to identify the erratic behavior of the discharge current as due to homoclinic chaos related to the Shilnikov scenario. It is worth mentioning that although the Shilnikov scenario has a solid mathematical background [3,4], it has only been previously observed in chemical reactions like the Belousov-Zhabotinskii reaction [5] and in some lasers with saturable absorbers [14].

The experiment consists of measuring the current through the glow discharge in Fig. 1. Convenient control parameters are either the source voltage $V_{S}$ or the gas pressure. We describe here what happens when keeping the pressure constant $(2 \mathrm{mbar})$ while changing $V_{S}$. For $V_{S}<500 \mathrm{~V}$ the discharge is off. Above this threshold one sees current spikes with the form of a relaxation oscillation [15]. Initially the peaks are widely spread and erratically distributed in time (i.e., there is a jitter). Their amplitude is of the order of $285 \mu \mathrm{A}$ while between peaks the current is essentially zero. By further increasing $V_{S}$ the time separation between successive peaks decreases and the oscillation assumes a perfectly regular shape in time. This occurs with the oscillations showing little change in their peak amplitudes as $V_{S}$ was increased. A typically observed pattern for the oscillation in this re- 


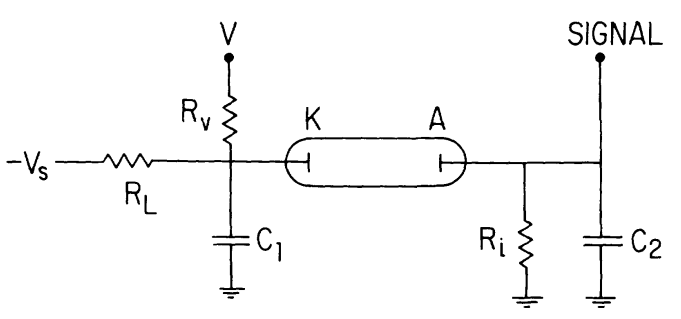

FIG. 1. Schematic view of the experiment. $R_{\mathrm{r}}=10 \mathrm{~m} \Omega$, $R_{L}=900 \mathrm{k} \Omega, R_{i}=2 \mathrm{k} \Omega, C_{1}=180 \mathrm{pF}$, and $C_{2}=5600 \mathrm{pF}$. The cylindrical brass electrodes are $11 \mathrm{~mm}$ apart, having diameters $12 \mathrm{~mm}$ (cathode) and $19.2 \mathrm{~mm}$ (anode). They are enclosed in a glass cell of $23.8 \mathrm{~mm}$ internal diameter with a flow of commercial grade argon at 2 mbar. The current is measured by the voltage drop through $\boldsymbol{R}_{\boldsymbol{i}}$.

gime is shown in Fig. 2(a), where we present the time evolution of the oscillations (left) together with the corresponding phase portrait (right). The phase portraits are obtained from phase-space trajectories reconstructed using the time-delay method from signals digitalized with a sampling time of $4.3 \mu \mathrm{s}$ and 16000 data points storage area. A projection in the 3D space $X=I(t), Y=I(t$ $+\tau), Z=I(t+2 \tau)$ with $\tau=12.9 \mu \mathrm{s}$ is shown in the figure. There are several criteria [16] for an adequate choice of the delay time $\tau$. Here we took empirically $\tau$ as $\sim \frac{1}{6}$ or $\frac{1}{7}$ of the period of a small-amplitude oscillation in the observed signal.

Raising $V_{S}$ one sees the bifurcation sequence illustrated in Fig. 2. First, the initial $P^{(0)}$ oscillation [Fig. 2(a)] bifurcates into a $P^{(1)}$ pattern [Fig. 2(b)]. By increasing $V_{S}$ the amplitude of the small-amplitude oscillation diminished continuously [Fig. 2(c)] until the occurrence of the second bifurcation: a period doubling of $P^{(1)}$ into $2 P^{(1)}$, as shown in Fig. 2(d). Further period doublings were not observed due to limitations on the resolution of changes in $V_{S}$. Thus, after $2 P^{(1)}$ we see the first chaotic window $C^{(1)}$, illustrated in Fig. 2(e). Increasing $V_{S}$, the chaotic oscillation $C^{(1)}$ suddenly stabilizes into a periodic $P^{(2)}$ pattern [Fig. 2(f)]. The next few bifurcations [Figs. $2(\mathrm{~g})-2(\mathrm{~m})]$ follow the same aforementioned sequence of events. Raising $V_{S}$ the amplitude of the $n$ smallamplitude oscillations in the $P^{(n)}$ pattern decreases until a $2 P^{(n)}$ period doubling occurs. After that, the oscillation turns into a $C^{(n)}$ chaotic one until it suddenly jumps into a $P^{(n+1)}$ periodic pattern, and so on. We have repeatedly observed this sequence up to the $P^{(4)}$ pattern [Fig. $2(\mathrm{~m})$ ]. After that, a chaotic pattern followed [Fig. 2(n)] and thereafter the oscillation drastically changed its shape, "phase transition"; i.e., there is a change from a mixedmode oscillation to a nearly $2 P$ sinusoidal oscillation [Fig. $2(0)]$. This oscillation underwent a reverse period doubling [Fig. 2(p)] and by further raising $V_{S}$ the resulting $P$ oscillation only diminished its amplitude until $V_{S}=673 \mathrm{~V}$ where it disappeared, remaining just a pure dc current.

The bifurcation sequence of Fig. 2 fits very well the APC sequence of a homoclinic chaos scenario $[10,11]$.

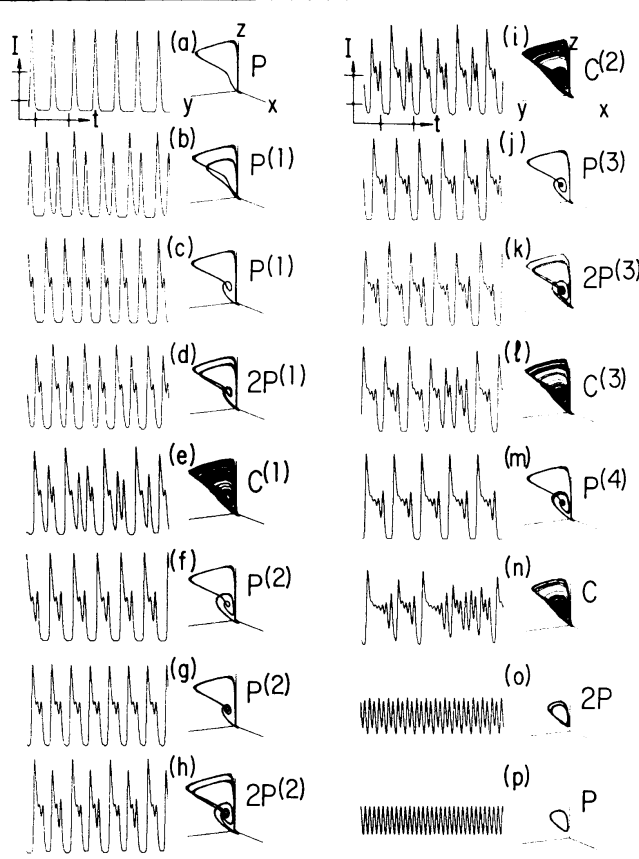

FIG. 2. Time evolution and reconstructed phase space of the current $I(t)$ flowing a dc-excited glow discharge in argon (see text). The current and time intervals shown correspond to 100 $\mu \mathrm{A}$ and $500 \mu \mathrm{s}$, respectively. In (a), $P=P^{(0)}$.

Further, the widths of periodic $P^{(n)}$ windows get smaller as $n$ increases. This fact is also in close agreement with the homoclinic chaos scenario [14] and explains why experimentally it is not possible to observe higher $n$ 's in an APC sequence: The width of the $P^{(n)}$ window and of the experimental noise may be of the same order. Hence, only a chaotic pattern is observable.

Additional evidence of homoclinic chaos is obtained from the reconstructed phase-space portraits displayed on the right of Fig. 2. The general feature of the dynamics in phase space consists of trajectories reaching a small region, from where they begin to spiral away until escaping eventually by doing a large loop in the phase space, being finally reinjected very near to the spiraling region again. Such evolution corresponds to a scenario where a homoclinic system displays a bounded domain (the saddle invariant set) in phase space connected to a homoclinic orbit [8-11]. According to this scheme [11], the trajectories approach the invariant set (either a fixed point or a periodic orbit) along the stable manifold, stay in its neighborhood for a while, and then escape along the unstable manifold. But nonlinearities present in the system bend the outgoing trajectories as they approach the homoclinic orbit such that they are reinjected back into the proximities of the invariant set. This rich reinjection mechanism was identified quite early by Rössler as capable of producing complex dynamical behavior [17]. The time spent in the neighborhood of the invariant set depends on the reinjection coordinates. For a small change in these coordinates, large time fluctuations may occur. 
This sensitive dependence on initial conditions is a hallmark of chaotic dynamical systems.

Yet another way of characterizing homoclinic chaos is via Poincaré maps obtained from reconstructed phasespace portraits $[3,4]$. However, we will prefer to apply a procedure first introduced by Arecchi et al. [7] while investigating instabilities on a modulated laser: the return-time map, which is an experimentally very convenient tool for analyzing homoclinic chaos. Rather than looking at the reconstructed phase-space trajectory, the information is derived directly from the time evolution of the signal. The relevant feature of the chaotic dynamics extracted by a return-time map is the fluctuation in the time necessary for the system to leave and come back to the invariant set. To obtain a return-time map we define a constant threshold level $I_{t}$ parallel to the $t$ axis in Fig. 2. Every time the current intersects this threshold level (from below) we record the time interval $t$ that it takes until the next similar intersection. Arranging the data in a plot $t_{i} \times t_{i+1}$ produces the return-time map. Figures 3 (a) and 3(b) show maps obtained for the chaotic signals shown in Figs. 2(e) and 2(i), respectively. These maps display a multibranched structure which evidences strong time fluctuations in the dynamics of the discharge. At the origin of these fluctuations are two distinct factors. One is the number of times the system turns around the invariant set (which corresponds to the number $n$ of small-amplitude oscillations in the $C^{(n)}$ pattern). This factor is responsible for the discontinuities in the branches of the map. The other factor is the time the system takes along the reinjection loop (i.e., the duration of the large-amplitude oscillation in the $C^{(n)}$ pattern). The closer the system is to the homoclinic orbit, the greater the time necessary to return to the invariant set; in other words, the distance of the system from homoclinicity determines the time spent in the reinjection loop. This increase in time is an evidence of homoclinic behavior and, in order to better use this evidence, from the time durations of the successive reinjection loops we construct a return map similar to that proposed by Papoff and coworkers [18] in their investigation of homoclinic chaos in a laser with saturable absorber: the "time-of-flight" re-

(a)

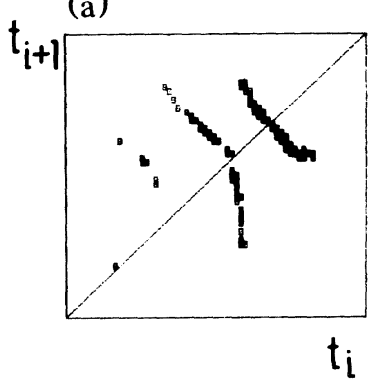

FIG. 3. Multibranched structure on return-time maps obtained from the hesitating signals in (a) Fig. 2(e) and (b) Fig. 2(i). turn map. For this purpose one defines now upper and lower current thresholds $I_{u}$ and $I_{l}$. The thresholds are chosen such that $I_{l}$ is slightly lower than the lowest excursion of the small-amplitude oscillations while $I_{u}$ is somewhat larger than the highest of the small-amplitude oscillations. The time necessary for the current to go from $I_{l}$ (from below) to $I_{u}$ (from above) is defined as the time of flight $\tau$ : It is essentially the duration of the reinjection loop. From them we construct the time-of-flight return maps $\tau_{i} \times \tau_{i+1}$, given in Figs. 4(a) and 4(b) again for the chaotic signals in Figs. 2(e) and 2(i), respectively. It is remarkable that the maps are still multibranched. A simple interpretation can be given to these branched structures. First, we notice that there is a correlation between the time of flight and the number of smallamplitude oscillations in the current of the discharge. A large (small) time of flight is followed by a higher (lower) number of small oscillations around the invariant set. This relation is reflected in the map by the fact that each branch may be identified with the number $n$ of small-amplitude oscillations as is shown in Fig. 4. Thus the branches in the map are labeled from left to right with increasing $n$. Continuing this sequence one should reach, in principle, a situation where the rightmost branch in the map corresponds to an infinite time of flight having $n=\infty$ associated to it. In this case, the trajectory would spend an infinite time approaching the invariant set along the reinjection loop whereas the infinite number of turns around the invariant set implies that it would take an infinite time to leave the invariant set. To sum up, we would have an orbit biasymptotic to the invariant set for $t \rightarrow \pm \infty$, matching exactly the definition of a homoclinic orbit [3].

The presence of a homoclinic orbit could also be determined from a current intensity return map. This map is obtained from the reconstructed phase portrait through a Poincaré section as shown elsewhere [19]. When the system is near homoclinicity such a map should also be multibranched, similar to the time-of-flight return map in Fig. $4[18,20]$. Indeed, it may be shown [18] that with an (a)

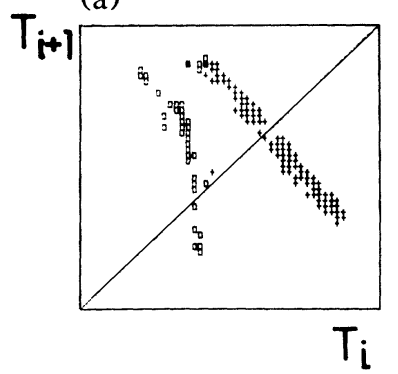

(b)

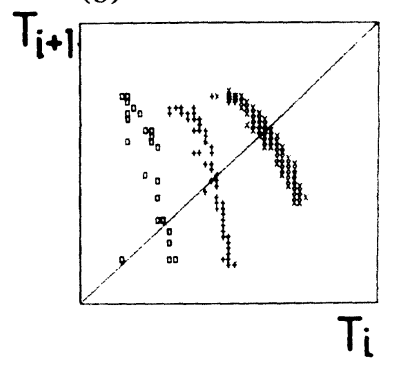

FIG. 4. Multibranched structure on time-of-flight maps obtained from the hesitating signals in (a) Fig. 2(e) and (b) Fig. 2(i). Squares correspond to $n=0$, plusses to $n=1$, and crosses to $n=2$. 
adequate choice of the threshold levels $I_{l}$ and $I_{u}$ there is a complete equivalence between the time-of-flight return map and the intensity return map. We have verified this equivalence in our system [19]. From the multibranched current return map one may also infer quasihomoclinic behavior using a symbolic dynamics approach. Further, one can also characterize the low-dimensional chaotic behavior in the discharge by calculating Lyapunov exponents. These results are presented elsewhere [19].

In conclusion, we have reported the time evolution and return maps associated with the current of a lowtemperature plasma medium, namely, a glow discharge. The evidence gathered indicates that the observed dynamics is closely related to a Shilnikov homoclinic chaos scenario. We showed that time-of-flight maps can be efficiently used to characterize homoclinic behavior directly from the definition of a homoclinic orbit. The present paper reports the first observation of homoclinic chaos in a plasma medium. As far as we know, the only previous experimental observations of this interesting and elusive phenomenon were done in chemical reactions and in lasers.

We thank Professor Ennio Arimondo, Università di Pisa, Italy, for many discussions during his one-month stay in our laboratories. T.B. and J.A.C.G. thank also E. Arimondo, F. Papoff, and B. Zambon for sharing with us their experience on the investigation of homoclinic chaos during our recent visit to Pisa. J.A.C.G. is a senior research fellow of the CNPq (Brazil). A preliminary report of this work was presented during the XII ENFMC in Caxambu, Brazil.

(a) Present address for 1992: Laboratory for Plasma Research, University of Maryland, College Park, MD 20742.

[1] See any book about chaos, e.g., H. G. Schuster, Deterministic Chaos. An Introduction ( $\mathrm{VCH}$ Verlagsgesellschaft, Weinheim, 1988), 2nd ed.

[2] H. L. Swinney, Physica (Amsterdam) 7D, 3 (1983).

[3] S. Wiggins, Global Bifurcations and Chaos-Analytical Methods (Springer-Verlag, New York, 1984).
[4] J. Guckenheimer and P. J. Holmes, Non Linear Oscillations, Dynamical Systems and Bifurcations of Vector Fields (Springer-Verlag, New York, 1984).

[5] F. Argoul, A. Arneodo, and P. Richetti, J. Chim. Phys. 84, 1367 (1987), and several of the 118 references therein.

[6] D. Hennequin, F. de Tomasi, B. Zambon, and E. Arimondo, Phys. Rev. A 37, 2243 (1988); D. Dangoisse, A. Bekkali, F. Pappof, and P. Glorieux, Europhys. Lett. 6, 335 (1988).

[7] F. T. Arecchi, A. Lapucci, R. Meuccci, J. A. Roversi, and P. H. Coullet, Europhys. Lett. 6, 677 (1988).

[8] L. P. Shilnikov, Math. SSSR Sbornik 10,91 (1970).

[9] N. K. Gavrilov and L. P. Shilnikov, Math. SSSR Sbornik 19, 139 (1973).

[10] P. Glendinning and C. Sparrow, J. Stat. Phys. 35, 645 (1984); P. Gaspard, R. Kapral, and G. Nicolis, J. Stat. Phys. 35, 697 (1984).

[11] P. Gaspard and X. J. Wang, J. Stat. Phys. 48, 151 (1987).

[12] T. Braun, J. A. Lisboa, R. E. Francke, and J. A. C. Gallas, Phys. Rev. Lett. 59, 613 (1987).

[13] P. Y. Cheung and A. Y. Wong, Phys. Rev. Lett. 59, 551 (1987); Q. L. Wang et al., Phys. Rev. Lett. 63, 163 (1989).

[14] See C. O. Weiss and R. Vilaseca, Dynamics of Lasers (VCH Verlagsgesellschaft, Weinheim, 1991), Chap. 6, for an account on the very recent progress on homoclinic behavior in lasers.

[15] Y. G. Zhakharenko and V. E. Privalov, Zh. Tekh. Fiz. 41, 553 (1971) [Sov. Phys. Tech. Phys. 16, 429 (1971)].

[16] Th. Buzug, T. Reimers, and G. Pfister, Europhys. Lett. 13, 605 (1990), and references therein.

[17] O. E. Rössler, Z. Naturforsch. 31a, 259 (1976); Ann. N.Y. Acad. Sci. 316, 376 (1979).

[18] F. Papoff, A. Fioretti, and E. Arimondo, Phys. Rev. A 44, 4639 (1991); F. Papoff, A. Fioretti, E. Arimondo, and N. B. Abraham, in Measures of Complexity and Chaos, edited by N. B. Abraham, A. M. Albano, A. Passamante, and P. E. Rapp (Plenum, New York, 1990), p. 309.

[19] T. Braun, J. Lisboa, and J. A. C. Gallas (to be published); T. Braun, Ph.D. thesis, Universidade Federal do Rio Grande do Sul (in Portuguese) (unpublished).

[20] M. Lefranc, D. Hennequin, and D. Dangoisse, J. Opt. Soc. Am. B 8, 239 (1991). 